\title{
De la nasse au filet
}

Stratégies halieutiques et modernisation dans le Rufiji (Tanzanie)

From barriers to nets: fisheries strategies and modernisation in Rufiji (Tanzania)

Jean-Luc Paul, Olivier Hamerlynck, Stéphanie Duvail et Kassim H.

Kindinda

\section{(2) OpenEdition}

Journals

Édition électronique

URL : https://journals.openedition.org/tc/7506

DOI : $10.4000 /$ tc. 7506

ISBN : 0248-6016

ISSN : 1952-420X

Éditeur

Éditions de l'EHESS

\section{Édition imprimée}

Date de publication : 1 mai 2015

Pagination : 234-257

ISBN : 9782713224874

ISSN : 0248-6016

\section{Référence électronique}

Jean-Luc Paul, Olivier Hamerlynck, Stéphanie Duvail et Kassim H. Kindinda, « De la nasse au filet », Techniques \& Culture [En ligne], 63 | 2015, mis en ligne le 22 décembre 2018, consulté le 29 septembre 2022. URL : http://journals.openedition.org/tc/7506 ; DOI : https://doi.org/10.4000/tc.7506 


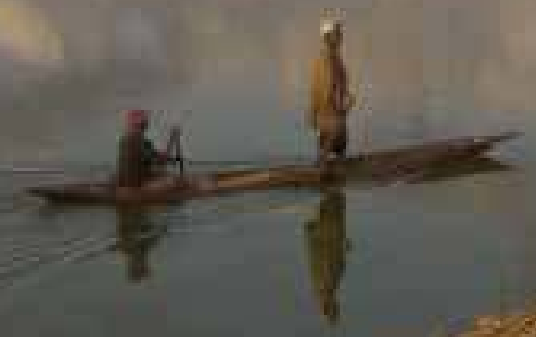




\section{DE LA NASSE AU FILET}

\section{Stratégies halieutiques et modernisation dans le Rufiji (Tanzanie)}

Depuis longtemps, les agronomes africanistes ont questionné les catégories classiques de l'analyse de la prise de décision technique des agriculteurs et remis en cause leur applicabilité aux paysans de l'Afrique subsaharienne et du Sud en général (de Schlippé, 1956; Milleville, 1974 et 1976). Stimulés par leurs échanges avec la géographie rurale africaine (Blanc-Pamard \& Milleville, 1985 par exemple) mais aussi d'autres disciplines (économie, anthropologie), ils ont élaboré un cadre théorique et méthodologique articulant aux contraintes écologiques et agro-techniques la diversité des rationalités économiques des producteurs déterminées par des cadres socio-économiques et des dynamiques historiques spécifiques (Dufumier, 1985). L'approche proposée par l'école française d'agriculture comparée est sans doute la forme la plus aboutie de ce cadre (Cochet, 2011). Au croisement de ces recherches et de l'anthropologie économique, le présent travail s'inscrit dans un programme de recherche sur les stratégies d'utilisation des ressources naturelles par les communautés locales des basses vallées d'Afrique de l'Est. L'économie de ces communautés est fondée sur la complémentarité des activités agricoles, des activités halieutiques et de l'exploitation des ressources forestières (Hamerlynck, \& al. sous presse). Depuis un siècle, cette complémentarité a été mise à mal par des politiques coloniales et postcoloniales qui ont considérablement réduit l'accès des communautés locales aux ressources naturelles ${ }^{1}$. Parallèlement, leur insertion forcée à l'économie de marché s'est faite dans des conditions d'échanges globalement défavorables², s'opposant à une quelconque accumulation qui aurait permis la modernisation des systèmes productifs. Bien qu'ici comme ailleurs les sociétés traditionnelles soient en involution ${ }^{3}$, leur relative résilience est remarquable. Une partie de cette résilience repose sur la double capacité, d'une part, 
de réaménager la complémentarité entre activités et, d'autre part, d'inventer ou d'adopter des solutions techniques compatibles avec l'absence d'accumulation. Dans cet article, nous nous intéressons à la substitution, qui a lieu à la fin des années 1970 dans la plaine inondable du Rufiji (Tanzanie), des pêches traditionnelles à la nasse, pratiquées à pied saisonnièrement lors de la crue annuelle, par les pêches au filet embarquées (pirogues monoxyles) dans les lacs, pratiquées elles tout au long de l'année. Nous nous interrogeons sur les déterminants de cette substitution. En questionnant l'hypothèse techniciste de l'automaticité du gain de productivité des techniques modernes vis-à-vis des techniques traditionnelles, nous proposons de dénouer l'écheveau des possibles causalités et de rendre au processus de modernisation sa dimension sociale ${ }^{4}$.

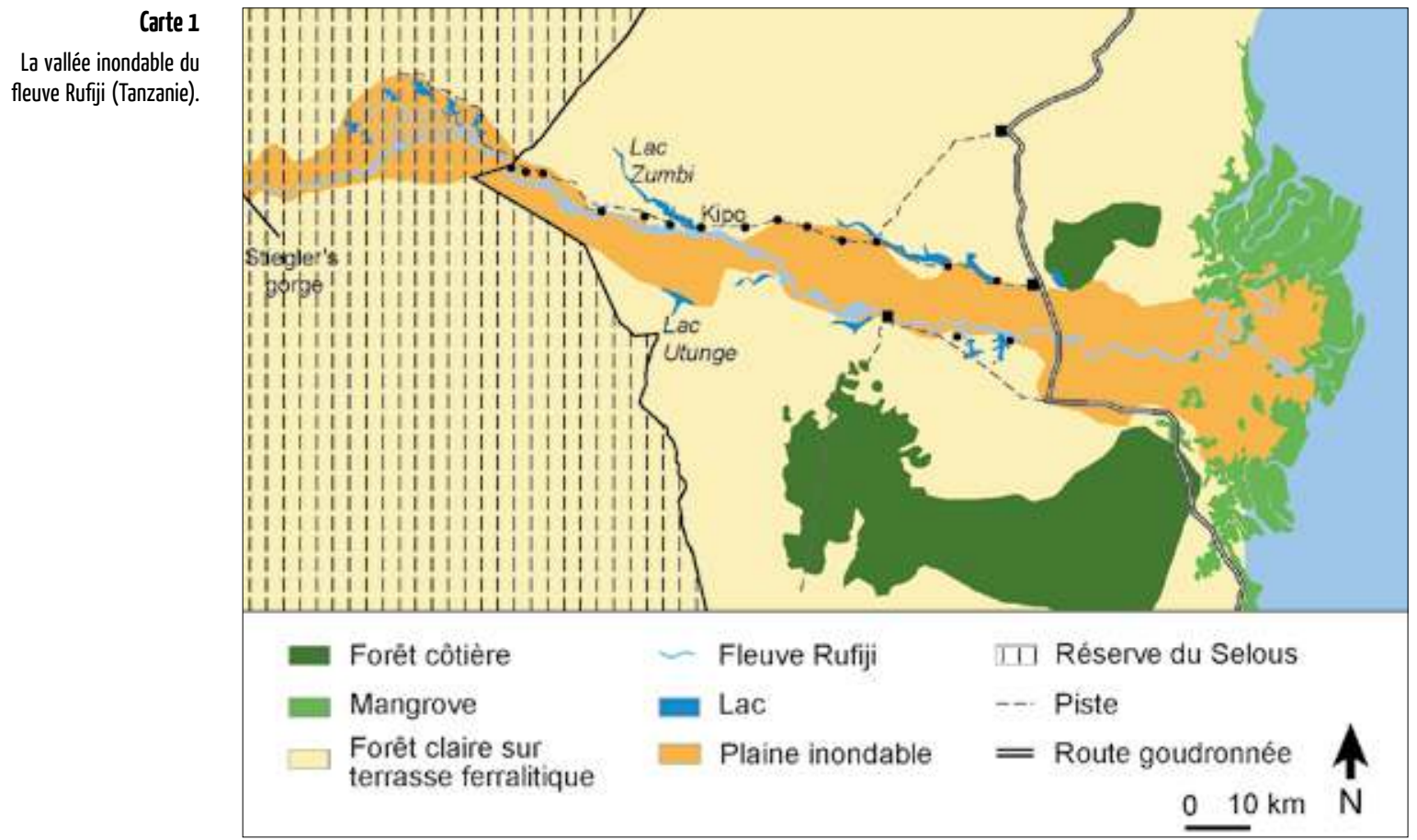

\section{Techniques halieutiques modernes et traditionnelles contemporaines}

Les variations saisonnières et annuelles du débit du fleuve Rufiji sont importantes: pour une moyenne de $850 \mathrm{~m}^{3} / \mathrm{s}$, le débit d'étiage est de l'ordre de $600 \mathrm{~m}^{3} / \mathrm{s}$ tandis que l'inondation de la plaine se produit autour du mois de mai pour des débits supérieurs à $2500 \mathrm{~m}^{3} / \mathrm{s}$, 




soit deux années sur trois pour la période 1956-2012. Elle couvre de larges superficies pour des débits supérieurs à $3500 \mathrm{~m}^{3} / \mathrm{s}$, soit une année sur deux pour la période 19561984 mais beaucoup plus rarement depuis. L'inondation par les eaux du fleuve Rufiji est la principale source d'alimentation de tous les réservoirs hydrologiques de la zone, qu'ils soient permanents (lacs) ou temporaires (mares, marécages, etc.) (Duvail \& al. 2013). Outre les alluvions et les remaniements micro-topographiques qu'elle engendre, la crue apporte avec elle une population de poissons adultes venus frayer et/ou se nourrir. Les poissons se déplacent en suivant les chenaux qui partent du fleuve vers les réservoirs. En fonction du courant, ils les parcourent dans les deux sens. Beaucoup de ces individus sont piégés dans les réservoirs au moment de la décrue, alors que les chenaux s'assèchent. Hamerlynck \& al. (2011) ont montré que le renouvellement de la ressource halieutique des lacs dépend avant tout du restockage assuré par la crue.

À partir d'observations directes, du suivi quotidien d'un échantillon de cinq pêcheurs (du 10 août 2007 au 30 mars 2010) et d'entretiens, tous conduits dans le village de Kipo, nous avons listé et caractérisé les différentes techniques de pêche utilisées en ce début du $\mathrm{xxI}^{\mathrm{e}}$ siècle dans la partie occidentale de la plaine inondable du Rufiji. Le village de Kipo est situé sur la terrasse ferralitique qui surplombe la rive septentrionale du fleuve (carte 1). Il est également adossé au lac Zumbi, lui-même séparé du fleuve par un seuil de quelques centaines de mètres de large. Sur l'autre rive du fleuve s'étend, sur plusieurs kilomètres de large, la plaine inondable. Elle accueille les parcelles des cultures pluviales (maïs) et inondables (riz) des villageois ainsi que de nombreux réservoirs d'eau naturels pour la plupart temporaires (mares, marécages et un lac). À une cinquantaine de kilomètres de Kipo, en amont, un delta intérieur du fleuve abrite de nombreux lacs poissonneux situés au sein de la Réserve du Selous et dont l'accès est interdit aux pêcheurs. Poussés par la 
Tableau 1

Répartition des jours pêchés en fonction du lieu de pêche (légal)

et de la technique

(suivi quotidien sur 30 mois -août 2007 à mars 2010- de 5 pêcheurs du village de Kipo). nécessité, certains hommes de Kipo y pêchent régulièrement au risque de leur vie. Cette pêche illégale, mais économiquement incontournable pour les familles dont un enfant poursuit des études secondaires, a également été caractérisée par de nombreuses enquêtes (Paul, Duvail \& Hamerlynck, 2011).

Les suivis montrent que la pêche au filet dans les lacs domine très largement les activités halieutiques. Nous tenant uniquement aux lieux légaux de pêche, la pêche au filet sur le lac Zumbi regroupe 88,8 \% des jours pêchés 5 .

\begin{tabular}{|l|c|c|l|}
\hline Lieu & $\begin{array}{c}\text { Echantillon } \\
\text { de 5 pêcheurs }\end{array}$ & $\begin{array}{c}\text { Pêcheur le } \\
\text { plus diversifié }\end{array}$ & \multicolumn{1}{|c|}{ Type de pêche } \\
\hline Lac Zumbi & 88,8 & 81,3 & Filet uniquement \\
\hline Fleuve Rufiji & 6,9 & 7 & Filet, ligne simple et palangre \\
\hline Plaine inondable & 3,2 & 11,7 & Nasse, panier et filet \\
\hline Non renseigné & 1,1 & 0 & - \\
\hline
\end{tabular}

La fréquentation des autres lieux de pêche (fleuve et réservoirs de la plaine inondable) est faible, soit un peu plus de 10 \% des jours pêchés dans les lieux légaux. Elle est associée à la mise en œuvre d'autres techniques comme l'utilisation de lignes ou de nasses.

$\begin{gathered}\text { Diverses pêches } \\ \text { dans la plaine inondable et dans le fleuve } \\ \text { (panier, ligne simple ou palangre, filet fixe) }\end{gathered}$
$\begin{gathered}\text { Pêine inondable } \\ \text { et paradières sur les } \\ \text { chenaux lac-fleuve } \\ \text { et palangre } \\ (5,8 \%)\end{gathered}$

Les différentes zone de pêche et les techniques associées dans la partie occidentale de la plaine inondable du Rufiji (Tanzanie) au début du xxı e siècle (NB : 1,1\% des situations de pêche n’ont pas été renseignés).(Fig. 1) 
Tableau 2

Principales caractéristiques des techniques de pêche pratiquées par les populations de la plaine du Rufiji au début du xxl e siècle (2007-2009 pour les valeurs monétaires).
La technique la plus utilisée est le filet fixe (tega). Une paire de pêcheurs embarquée dans une pirogue pose le filet à la tombée de la nuit, retourne se reposer sur la berge, relève le filet une première fois au milieu de la nuit puis une seconde fois au petit matin. Ce travail nocturne peu exigeant permet un travail agricole diurne, il est techniquement simple et nécessite un filet de taille moyenne ${ }^{6}$. Viennent ensuite les techniques de senne embarquée qui se pratiquent essentiellement de jour. Le njenje se pratique à une seule pirogue accueillant une paire de pêcheurs tandis que le juya exige deux pirogues (4 pêcheurs au total). Dépense physique, technicité et taille du filet sont sensiblement plus élevées que dans le cas précédent et le travail agricole diurne n'est guère envisageable le jour de pêche. La technique juya est illégale mais tolérée par les autorités villageoises et encouragée par les acheteurs en raison de sa forte productivité. Enfin, la pêche à l'épervier, également illégale, est aussi pratiquée sur le lac Zumbi mais le lancer n'est pas encore maîtrisé par les pêcheurs de Kipo. C'est un pêcheur allochtone, embarqué sur une pirogue conduite par un pêcheur local, qui l'effectue.

Les autres techniques de pêche occupent une place marginale. On pêche à l'hameçon dans le fleuve (ligne simple ndowano ou palangre ndowano za kutega), au panier (kisi) dans les mares résiduelles et à la nasse (kijumba) dans les chenaux qui relient le fleuve aux lacs. Paniers et nasses sont confectionnés par le pêcheur à partir de matériaux locaux (chaume de roseaux et folioles de palme). La pêche au panier représente moins de $1 \%$ des jours pêchés de notre échantillon. Elle est très saisonnière: il faut attendre que le poisson soit concentré dans une très faible profondeur d'eau par l'assèchement des mares formées par la crue, dans la plaine ou sur les bancs sablo-argileux du lit fluvial. La pêche à la nasse et aux paradières représentent environ $2 \%$ des jours pêchés. Elle se pratique essentiellement en début et en fin de crue, lorsque les chenaux de circulation de l'eau sont suffisamment étroits pour être barrés par des paradières.

\begin{tabular}{|c|c|c|c|c|c|c|c|c|c|c|c|}
\hline \multirow{2}{*}{ Z } & & \multirow{2}{*}{ 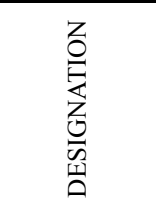 } & \multirow{2}{*}{  } & \multirow{2}{*}{ 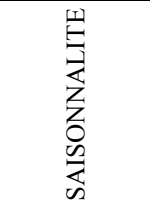 } & \multirow{2}{*}{ 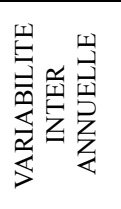 } & \multirow{2}{*}{ 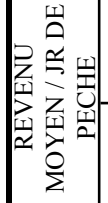 } & \multicolumn{2}{|c|}{$\begin{array}{c}\text { JOURS NECESSAIRES AU } \\
\text { BUDGET ANNUEL MOYEN } \\
\text { D'UN FOYER }\end{array}$} & \multirow{2}{*}{ 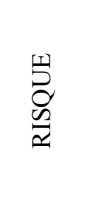 } & \multirow{2}{*}{ 占罟 } & \multirow{2}{*}{ 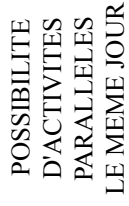 } \\
\hline & & & & & & & $\begin{array}{l}\text { Sans frais de } \\
\text { scolarisation }\end{array}$ & $\begin{array}{c}\text { Avec frais de } \\
\text { scolarisation } \\
\text { en secondaire }\end{array}$ & & & \\
\hline \multirow{7}{*}{ 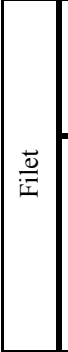 } & \multirow{2}{*}{ 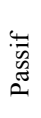 } & \multirow[b]{2}{*}{ Filet fixe } & Lac Zumbi & Peu marquée & Moyenne & 0,83 & 207 & 584 & \multirow[b]{2}{*}{ Faible } & \multirow[b]{2}{*}{ Légal } & \multirow[b]{2}{*}{ Oui } \\
\hline & & & $\begin{array}{l}\text { Plaine inondable et } \\
\text { lit mineur du fleuve }\end{array}$ & Marquée & Forte & 1,43 & 120 & 339 & & & \\
\hline & \multirow{5}{*}{ 偌 } & \multirow{3}{*}{$\begin{array}{c}\text { Senne } \\
1 \text { pirogue }\end{array}$} & Lac Zumbi & \multirow{2}{*}{ Peu marquée } & \multirow{5}{*}{ Moyenne } & 1,93 & 89 & 251 & Faible & Légal & Faible \\
\hline & & & Selous & & & 3,25 & 53 & 149 & Fort & Illégal & Non \\
\hline & & & Fleuve Rufiji & Marquée & & - & - & - & Faible & Légal & Faible \\
\hline & & $\begin{array}{c}\text { Senne } \\
2 \text { pirogues }\end{array}$ & \multirow[t]{2}{*}{ Lac Zumbi } & \multirow[t]{2}{*}{ Peu marquée } & & 2,45 & 70 & 198 & Moyen & Illégal & \multirow[t]{2}{*}{ Non } \\
\hline & & Epervier & & & & 2,47 & 69 & 196 & Moyen & Illégal & \\
\hline \multirow{2}{*}{ 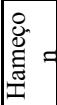 } & \multirow{2}{*}{ 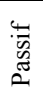 } & Ligne simple & \multirow{2}{*}{$\begin{array}{l}\text { Lac Zumbi et } \\
\text { fleuve Rufiji }\end{array}$} & \multirow{2}{*}{ Marquée } & \multirow{2}{*}{ Moyenne } & \multirow{2}{*}{1,68} & \multirow{2}{*}{102} & \multirow{2}{*}{288} & \multirow{2}{*}{ Faible } & \multirow{2}{*}{ Légal } & Faible \\
\hline & & Palangre & & & & & & & & & Oui \\
\hline \multirow{4}{*}{$\begin{array}{l}0 \\
\tilde{D} \\
\tilde{z} \\
z\end{array}$} & \multirow{2}{*}{ 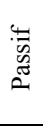 } & \multirow{2}{*}{$\begin{array}{c}\text { Palissade à } \\
\text { nasse simple } \\
\text { ou double }\end{array}$} & $\begin{array}{c}\text { Chenaux fleuve/lac } \\
\text { Zumbi }\end{array}$ & & & & & & Faible & & Oui \\
\hline & & & $\begin{array}{c}\text { Chenaux de la } \\
\text { plaine inondable }\end{array}$ & Marquee & Forte & 1,95 & 88 & 249 & Moyen & Legal & Non \\
\hline & $\mathscr{Z}$ & $\begin{array}{l}\text { Panier } \\
\text { percé }\end{array}$ & $\begin{array}{c}\text { Plaine inondable et } \\
\text { du lit mineur du } \\
\text { fleuve }\end{array}$ & Très marquée & Forte & 1,77 & 97 & 274 & Nul & Légal & Non \\
\hline & & renversé & $\begin{array}{l}\text { Bords du lac } \\
\text { Zumbi }\end{array}$ & & & & & & Moyen & & \\
\hline
\end{tabular}


Les pêches à la nasse et au panier peuvent être qualifiées de pêches traditionnelles. Elles sont anciennes, présentes au moins depuis la période coloniale. Les enquêtes suggèrent qu'elles sont le reliquat d'un éventail de techniques naguère communes et tombées en désuétude en raison de l'adoption généralisée du filet. À quoi correspond cette évolution et quelles en sont les déterminants, les modalités et les conséquences socioéconomiques? Après une caractérisation rapide des techniques de pêche qui dominaient l'activité halieutique avant l'avènement du filet, nous avons tenté d'identifier les causes de leur marginalisation brutale à partir de l'exemple de la pêche à la battue.

\section{Les pêches traditionnelles désuètes: l'exemple de la pêche}

\section{à la battue}

Les techniques traditionnelles sont pratiquées en eau peu profonde où les pêcheurs se déplacent à pied. Elles utilisent des engins de capture fabriqués localement, le plus souvent passifs. On tresse des éléments (wando-nyando ou lutangamatanga), dont la taille approximative est d'une toise $^{3}$ de hauteur sur deux de longueur, selon les mêmes techniques que celles utilisées pour réaliser des éléments architecturaux des cases de champs

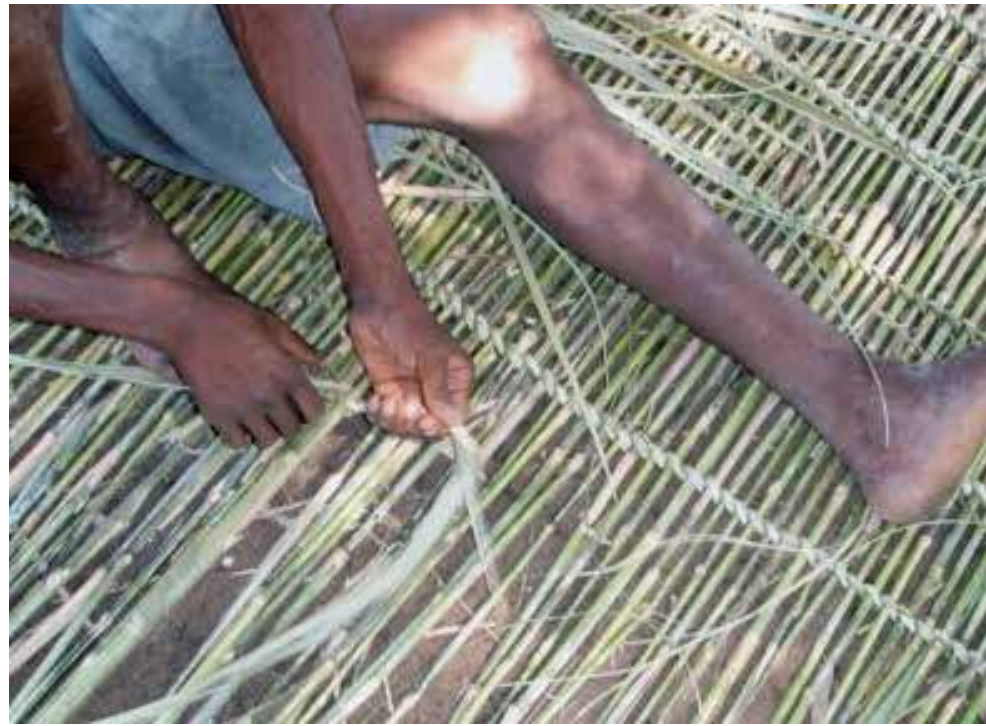

Haut: Timbinyu tresse un wando à l'aide de folioles du palmier doum (Hyphaene thebaica) et des chaumes de Phragmites mauritianus.

Bas: Au centre du chenal reliant le fleuve Rufiji au lac Zumbi durant la crue, deux nasses cordiformes ont été placées tête-bêche. Au premier plan, une première paradière joint la berge aux nasses. En arrière-plan, une autre paradière, ici cachée par les nasses, rejoint l'autre berge. 


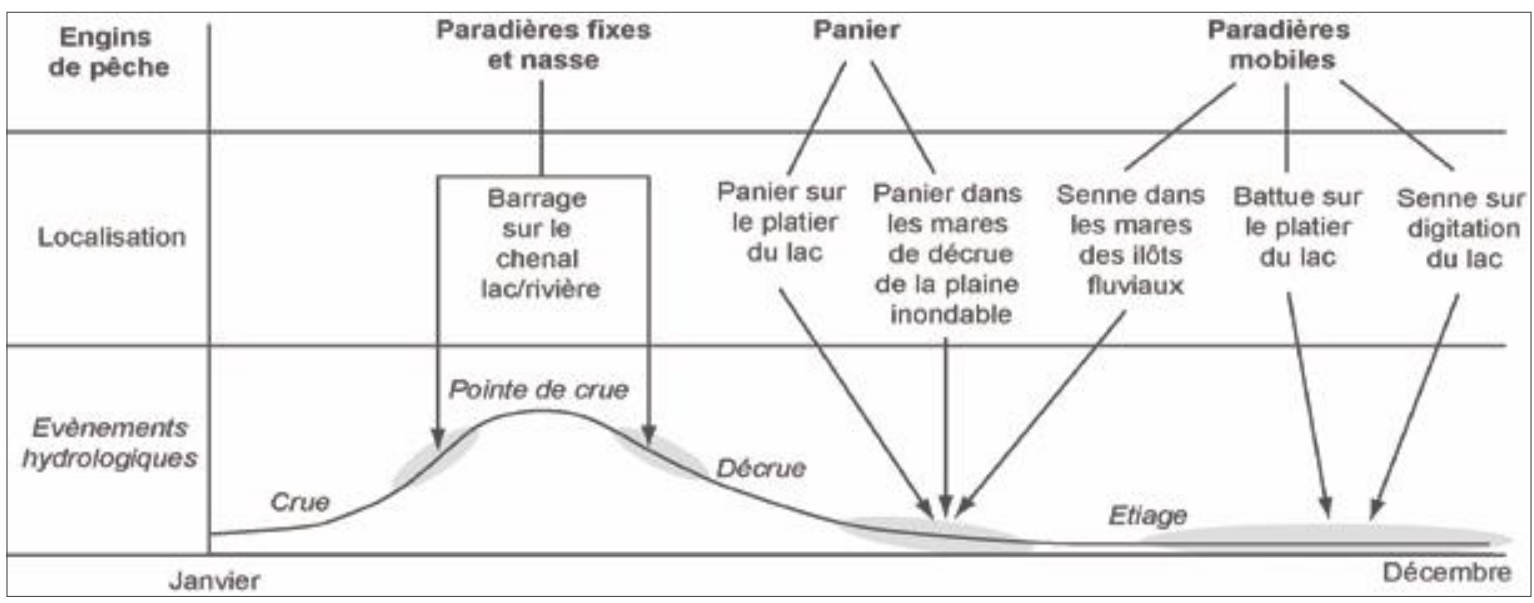

Figure 2

Les différentes techniques de pêche traditionnelles et les lieux et saisons associées dans la partie occidentale de la plaine inondable du fleuve Rufiji (Tanzanie).

(dungu-madungu) ou des récipients de stockage du riz (kitenge-vitenge). Ces éléments sont ensuite formés en grandes nasses cordiformes (kijumba-vijumba) ou aboutées pour former une paradière.

Les techniques traditionnelles utilisent la concentration naturelle du poisson, qu'il circule dans les étroits chenaux reliant le fleuve à la plaine ou au lac, qu'il soit piégé par la récession de l'eau dans des mares en voie d'assèchement ou encore qu'il se nourrisse sur les platiers du lac.

Dans presque tous les cas, des paradières sont utilisées, soit à la manière d'une senne (engin actif), soit comme un barrage qui force le poisson vers les nasses (engin passif). Une battue peut-être organisée dans des conditions que nous décrirons dans la suite du texte.

De toutes ces pêches, c'est la pêche à la battue qui reste dans les esprits des anciens comme la pêche par excellence. Voici dans quels termes Alawe Makuka se souvient d'une des plus fameuses pêches à la battue de sa jeunesse:

Figure 3

Déroulement de la pêche

à la battue dans le lac Zumbi (source: enquêtes auprès Alawe Salum Mkali et Ndumbo Sule, 2008).
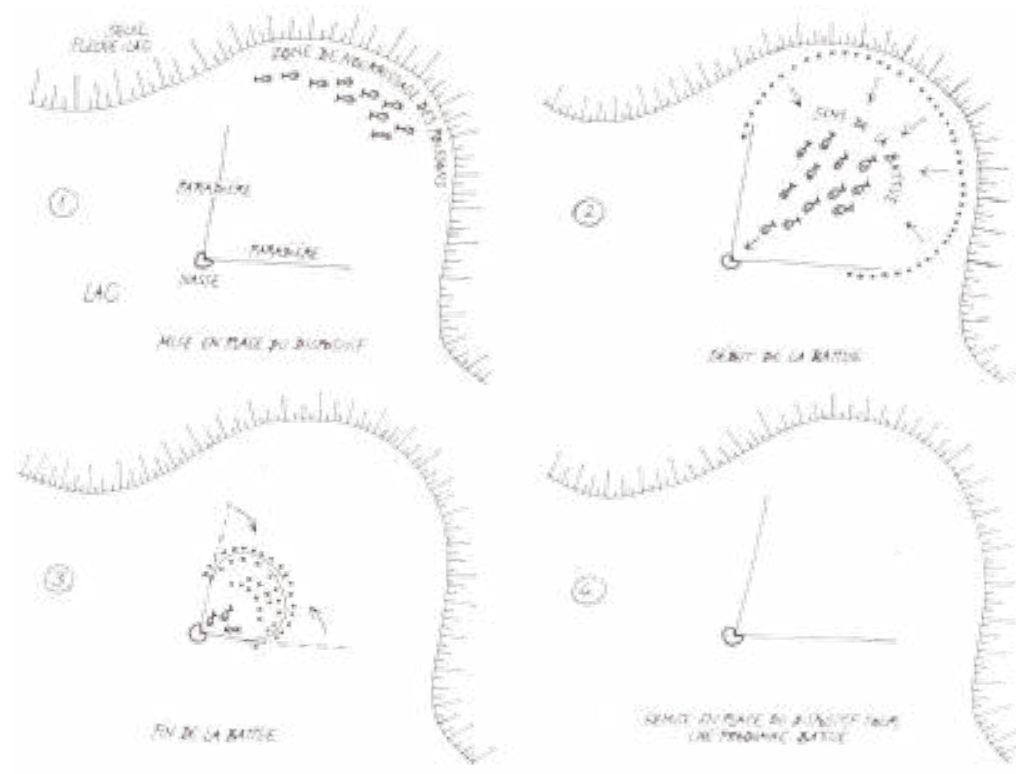
« En 1945, la famine était sévère. Il y avait un Européen appelé John Young. Il était le District Officer du Rufiji et il fut d'une grande aide. Mais nous, grâce au lac Zumbi, nous n'avons pas connu la disette. On a pêché énormément de poisson et nos voisins de l'intérieur des terres (...) venaient nous l'acheter. Des marchands sortaient également de Dar-es-Salaam. On vendait un poisson pour 15 ou 20 centimes de shilling de l'époque. 15 centimes! Et encore pour un pele (Citharinus congicus) gros comme ça (une quarantaine de $\mathrm{cm}$ de long ${ }^{8}$ ). Grâce à la pêche dans le lac, nous n'avons pas eu le tracas d'aller ici où là chercher de la nourriture ${ }^{9}$. En 1945, j'ai donc pêché énormément. C'était la disette et les anciens des hameaux de Mpande et Mgoholi commandèrent aux jeunes et aux autres anciens: allons pêcher! Ils réunirent les lignages, le lignage des Mbonde, celui des Malenda, celui des Lusonzo et les autres. Les gens commencèrent à tresser les palissades. Moi, j'étais sous l'autorité de Dogaga, l'ancien de notre lignage, les Mpingi. C'était notre dirigeant. On a coupé les roseaux, on les a tressés. Une fois qu'on eut fini de les tresser, les anciens se sont réunis.

- Les palissades sont-elles prêtes?

- Elles sont prêtes!

- Tel jour nous irons pêcher...

Ce sont les jeunes qui portèrent les palissades de la plaine inondable jusqu'au lac, de l'autre côté du fleuve. Dans mon lignage, les anciens étaient Msenge, Salum et Seifu Bora. Voilà pour ceux des anciens qui venaient de chez nous. Et nous, les jeunes, nous étions là pour faire le travail qu'on nous demandait de faire. Ceux qui venaient de chez nous, nous étions environ 5. D'autres venaient également avec 5 autres personnes, et une fois réunis on était une trentaine, mais tous sous les ordres des anciens.

- Faites ceci, faites cela! Vous les jeunes, mettezvous là!

Et ainsi de suite... On nous indiquait quoi faire, mais tous nous étions comme une seule chose sous les ordres des anciens. Il y avait bien quelques personnes qui voulaient pêcher pour eux-mêmes, à 2 ou 3. Ils pouvaient barrer un bras étroit du lac et poser leur nasse. Mais la plupart d'entre nous, nous étions ensemble au même endroit. Et même si nous étions 50, personne ne manquait de poisson (...) Nous pêchions à la battue. La palissade faisait une centaine de mètres de long. On installait la palissade de part et d'autre de la nasse (...) Les anciens connaissaient bien les poissons, ils pouvaient reconnaître l'espèce en fonction des remous. Ils se tenaient sur les berges, les poissons entraient sur le platier ${ }^{10} \mathrm{du}$ lac et quand ils l'avaient rempli, les anciens le savaient.

- Les poissons sont déjà entrés (...) Battez l'eau!

On battait l'eau, avec les bras, avec un bâton. Ça faisait un bruit comme celui de quelque chose qu'on pile dans le mortier: Toum! Toum! Toum! En même temps, on rapprochait les palissades jusqu'à les joindre. Les poissons qui étaient vers la berge sortaient pour se diriger vers le large. Ils entraient dans la nasse. Il n'y avait plus d'issue. Quand la nasse était pleine, on appelait une pirogue et on la chargeait. La pirogue se trouvait de l'autre côté de la palissade (vers le lac) (...) On faisait plusieurs battues, les palissades restaient longtemps en place, un mois voire deux, jusqu'à ce que les cordes du tressage se détériorent.

Il y avait des gens pour faire le partage, des gens choisis par les anciens.

- Tel jeune, tel autre, etc. Vous serez en charge du partage!

Il y avait du poisson en abondance. Ils formaient des tas en fonction de la taille des poissons. Il y avait 4 ou 5 tas. Les anciens étaient simplement assis.

- Combien sommes-nous?

- Nous sommes 30.

On faisait 30 lots. Ils commençaient à partager les gros poissons, 10 dans chacun des lots par exemple, jusqu'à 30 lots. Et ainsi de suite jusqu'à épuisement du stock. C'était comme ça qu'on partageait. Tu prenais ton lot, tu allais là-bas pour sécher le poisson. Si un revendeur venait tu pouvais vendre, comme bon te semblait. Chacun avait sa part. Pour ça, je ne dépendais pas de mon ancien, j'avais mon propre lot, bien que ce soit l'ancien qui nous avait donné l'ordre à la maison (d'aller pêcher). Il avait simplement donné l'ordre « sauvons-nous de la disette! » C'est comme quand il disait « allons cultiver! ». Une fois cultivé et récolté, il ne vient pas s'occuper des affaires de ta récolte et là-bas une fois les poissons pêchés, il ne se mêlait pas de tes affaires. »

Alawe Mkali (né au début des années 1920) 
Avant la généralisation de la pêche au filet, la pirogue monoxyle était avant tout un moyen de transport, y compris vers les lieux de pêche. Ici des villageois embarquent, au

petit matin, pour aller rejoindre leurs champs dans la plaine inondable.

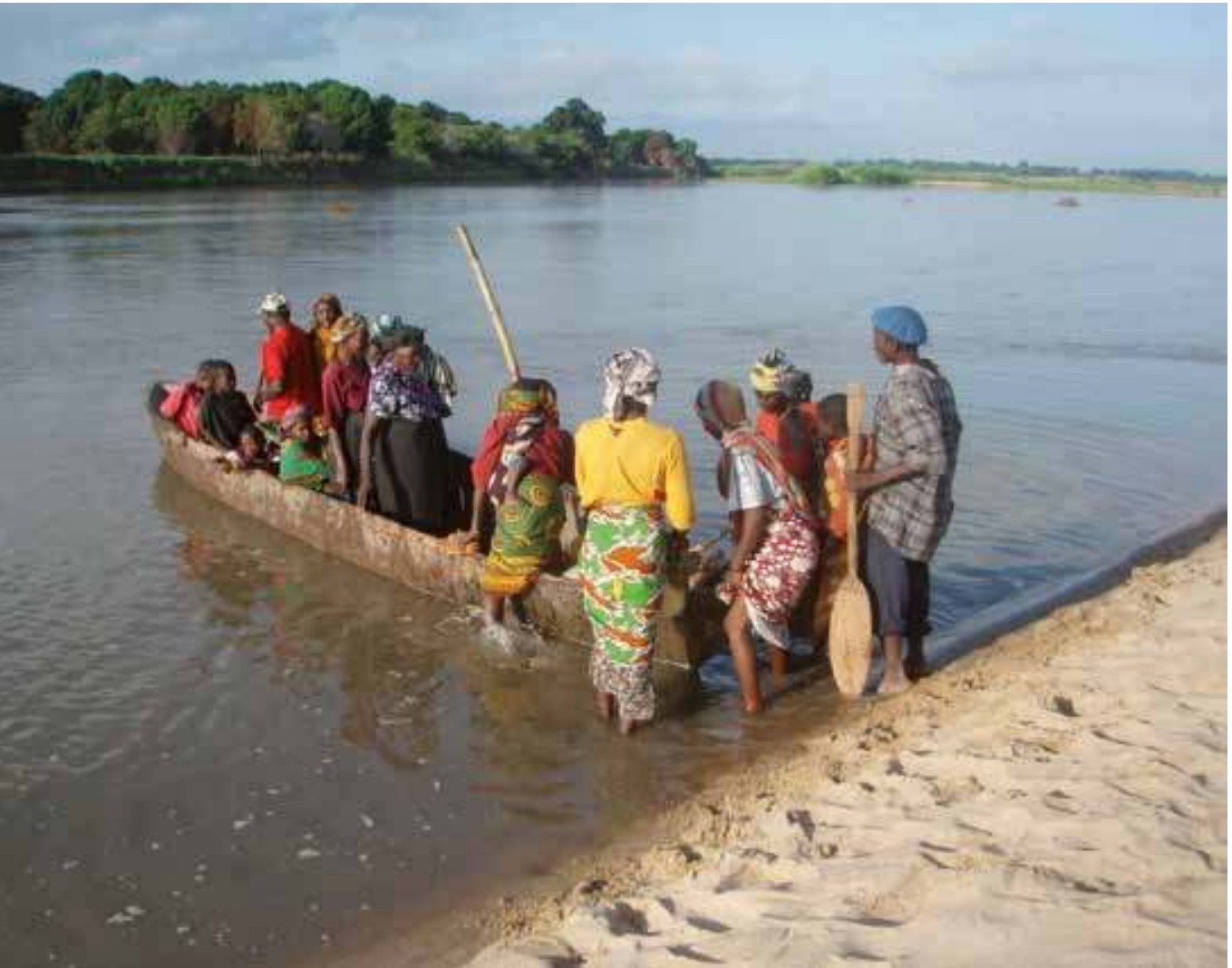

Le récit, très complet, décrit la technique de pêche, la répartition du produit, la vente aux populations environnantes, le rôle des lignages et de la coopération. La pêche à la battue sur les platiers du lac nécessite la coopération de plusieurs dizaines de personnes ${ }^{11}$ pour la confection d'une palissade pouvant excéder cent mètres et pour la battue en elle-même. Cette coopération mobilise les mêmes relations de parenté-affinité et de voisinage que les travaux agricoles mais ici élargies à plusieurs hameaux et à l'exclusion des femmes. Quelques différentiations s'esquissent entre les guetteurs qui jugent de l'opportunité de débuter la battue, les chasseurs de crocodile qui assurent la sécurité des rabatteurs, ceux qui pénètrent dans les nasses pour puiser les poissons et, enfin, les rabatteurs eux-mêmes. Il ne s'agit cependant pas d'une réelle spécialisation, chacun pouvant, avec plus ou moins d'habileté, assurer l'une ou l'autre des tâches. Le seul élément véritablement discriminant est l'appartenance générationnelle: tandis que les anciens dirigent les opérations, les cadets (adolescents et jeunes adultes célibataires, soit environ jusqu'à 30 ans) les réalisent. Et si ces derniers participent à la confection des palissades et à la battue, c'est à nouveau sous la supervision de leurs anciens reconnus comme les possesseurs de ces palissades. Enfin, la répartition du produit de la pêche semble équitable, mais nous verrons ce point remis en question par d'autres témoignages.

Les anciens parlent avec passion des pêches à la battue de leur jeunesse. Ils évoquent l'effervescence qui se manifeste à cette occasion et l'importance des prises. S'agit-il du discours nostalgique que tiennent certaines personnes âgées lorsqu'elles évoquent leur jeunesse ou peut-on leur accorder un certain crédit? On ne dispose malheureusement d'aucune source qui nous permettrait d'appréhender l'évolution de la pêche dans la zone au cours du xxe siècle. Cependant, la convergence des récits et l'absence de contradiction entre les différents informateurs plaident en faveur de leur fiabilité. Au-delà de cet élément de cohérence des discours, les récits restent compatibles avec les connaissances biologiques et écologiques contemporaines (par exemple, les indications de tailles et poids des individus pêchés ou les descriptions du requin du Zambèze, Carcharhinus leucas, et du poisson-scie, Pristis sp., aujourd'hui disparus de la zone). Les anciens décrivent aussi une meilleure connexion lac-fleuve, arguant que le chenal les reliant était régulièrement pâturé par les éléphants alors qu'il est aujourd'hui encombré des rebuts du nettoyage des parcelles attenantes et d'entretien de la piste qui l'enjambe. Enfin, ils insistent sur la saisonnalité de leur pêche et sa localisation aux seuls platiers du lac 
qui permettaient à un grand nombre de poissons d'échapper à la prise. La pêche à la battue était donc vraisemblablement une pêche particulièrement productive. Tout ceci rend d'autant plus problématique la question de la marginalisation des pêches à la nasse en général et de la disparition de la pêche à la battue en particulier. Comment donc et pourquoi une pêche si productive aura-t-elle finalement été abandonnée?

\section{Les causes de la marginalisation des techniques traditionnelles}

L'explication techniciste vulgaire des processus d'innovation technique repose sur l'idéologie du progrès, la modernité d'une technique la rendant ipso facto plus productive qu'une technique traditionnelle. En l'occurrence, la comparaison des pêches à la nasse et des pêches au filet ne conforte pas ce point de vue. La productivité journalière de la pêche à la nasse, telle qu'elle est encore aujourd'hui pratiquée, est strictement identique à celle de la plus productive des pêches au filet légales. Les pêches au filet illégales la surclassent dans ce domaine mais sont associées à de fortes contraintes spécifiques ${ }^{12}$. Au moment de l'adoption des techniques au filet, la comparaison était encore plus favorable à la pêche à la nasse car la taille des filets était réduite, leur confection laborieuse et ils étaient utilisés comme senne de plage ou comme filet fixe, en fait les techniques au filet les moins productives.

Il faut donc explorer plus avant les causes de l'adoption du filet en analysant les différentes implications techniques, économiques et sociales qui lui sont associées.

La pêche au filet permet de s'émanciper de la forte saisonnalité à laquelle sont soumises les pêches à la nasse ce qui améliore la productivité annuelle de l'activité halieutique. Les pêches à la nasse ne sont possibles qu'à deux conditions: la présence d'eaux de faible profondeur et la relative concentration du poisson. Ces conditions ne sont réunies que durant certaines périodes de la crue en de nombreux lieux dispersés dans la plaine inondable et à de plus rares emplacements localisés sur le seuil qui sépare les lacs du fleuve. Dans les lacs à proprement parler, c'est dans la seconde moitié de la saison sèche, au maximum de l'étiage, que la pêche à la battue est possible sur les platiers. Avec le filet, la pêche est possible, grosso modo, toute l'année. A contrario, la pêche au filet n'est pas possible en eaux peu profondes et sa pratique se concentre dans les lacs. Avec le passage au filet, il y a donc une extension dans le temps et une réduction dans l'espace des opportunités de pêche. Si, au lieu de la journée de pêche, on choisit l'année pour mesurer la productivité, même la pêche au filet la moins productive surclasse les pêches à la nasse. Ainsi, si la pêche à la nasse contemporaine est 3,6 fois plus productive par jour de pêche que la pêche au filet fixe (tableau 3); à l'année, la productivité s'inverse: le plus faible revenu annuel des pêcheurs suivis sur le lac Zumbi (260€) nécessiterait 93 jours de pêche à la nasse sur le chenal du lac Utunge tandis que la durée maximale possible n'excède pas deux mois...

La pêche au filet permet une émancipation relative de la crue ce qui améliore la productivité pluriannuelle des activités halieutiques (si les occurrences de sécheresse sont supérieures aux occurrences de fortes crues d'un débit supérieur à $3500 \mathrm{~m}^{3} / \mathrm{s}$, ce qui est le cas pour la période considérée). La crue est indispensable à l'activité halieutique mais ses caractéristiques ont 
une influence beaucoup plus imminente sur la pêche traditionnelle que sur la pêche au filet: sans crue, il n'y a pas de pêche dans la plaine. Par contre, la pêche au filet est possible en l'absence de crue. Dans un premier temps, elle la rend même plus productive par effet de concentration du poisson (la surface du lac diminue). C'est l'absence répétée et successive de crue qui entraîne une baisse de la productivité de la pêche au filet par diminution des stocks d'eau et de poissons. À léchelle pluriannuelle, la pêche au filet amortit le risque que l'absence de crue fait peser sur l'activité halieutique une année donnée.

La priorité accordée à un pas de temps particulier (pluriannuel, annuel ou journalier) dans l'élaboration des stratégies halieutiques villageoises dépend de la fonction économique de la pêche. Or, cette fonction a sensiblement évolué au cours de la période considérée, évolution qui traduit les changements des modalités d'articulation de l'économie villageoise au marché.

Au cours des deux décennies qui entourent l'indépendance (années 1950 et 1960) le pôle marchand de l'économie villageoise est essentiellement agricole ${ }^{13}$.

Les années de crue modérée, la vente des surplus de riz constitue l'essentiel du revenu monétaire. La récolte du riz absorbe l'essentiel du travail disponible et limite la surface semée en coton de décrue. La pêche dans la plaine inondable procure alors un revenu d'appoint au moment de la soudure ${ }^{14}$, plus ou moins marquée selon les résultats de la campagne agricole précédente. L'intérêt pour la pêche à la battue est réduit dans la mesure où les abondantes récoltes permettent de rester dans la plaine inondable et de se concentrer sur la préparation de la prochaine saison culturale.

Une crue importante qui détruit les cultures de riz entraîne la disponibilité de larges surfaces pour les cultures de décrue. Du coton et du maïs sont alors semés, l'un procurant le revenu monétaire, l'autre le vivrier. La pêche dans la plaine inondable joue alors un rôle clef, elle permet de faire face à une soudure particulièrement prolongée par le troc ou la vente. Par contre, les conditions sont défavorables à la pêche à la battue dans les lacs: persistance des hautes eaux et nécessité de rester dans la plaine pour assurer les travaux de mise en culture de décrue.

Les années de sécheresse (faibles précipitations et absence de crue) sont des années de crise. Les systèmes de culture dominants et l'activité halieutique ordinaire ne remplissent plus leurs fonctions. Des activités habituellement marginales et/ou opportunistes sont mises en œuvre: mise en culture des bancs fluviaux, mobilisation des ressources forestières, aide des membres urbanisés de la famille voire aide alimentaire gouvernementale si la sécheresse est très marquée. La pêche collective à la battue, pratiquée dans le lac en période de basses eaux, est alors d'un grand secours, comme ce fut le cas en 1945.

Ainsi, jusqu'alors, la pêche est une activité de soudure qui correspond à la période moyenne de crue ou, pour les années à très faible pluviométrie, à la saison sèche. C'est la productivité durant ces périodes-là, pour laquelle les techniques de pêche traditionnelle sont les plus performantes, qui importe alors.

À partir de la seconde moitié des années 1970 et jusqu'à la fin des années 1980, la situation économique des producteurs et de leurs familles se dégrade sensiblement sous la conjonction de facteurs interdépendants: décisions de politiques de développement (comme le contrôle accru des coopératives puis leur dissolution et leur remplacement par les agences parapubliques), dégradation des prix des produits d'exportation et de la situation géopolitique (dissolution de la East African Community en 1977, guerre contre l'Ouganda en 1978-1979) (Maghimbi 1992, Nindi 1992, Havnevik 1993, Bryceson 1996, Mpangala 1996, Mvungi \& Mwakyembe 1996, Sundet 1996, Biermann 1998, Bigsten \& 
Danielson 2001). En ce qui concerne le Rufiji, un événement central est la villagisation de 1968 qui va aboutir au bouleversement du système productif local ${ }^{15}$. Les populations, qui vivaient dans des hameaux dispersés de la plaine inondable, sont regroupées en villages installés à proximité des lacs de la terrasse ferralitique, sur la rive gauche du fleuve Rufiji. Les habitants des nouveaux villages se trouvent ainsi coupés de leur zone de culture habituelle. Répondant aux injonctions de l'administration du District, ils mettent en culture les sols ferralitiques qui rapidement se révèlent improductifs. Ils se tournent alors vers les dépressions argileuses des terrasses qui concentrent par ruissellement les eaux pluviales. Si elles sont plus productives, elles n'autorisent qu'une seule culture, le riz, et pour une unique récolte annuelle. Cependant, grâce à l'aide alimentaire de la première année d'installation, à une météorologie propice et à un environnement socioéconomique plutôt favorable, le bouleversement de l'organisation de la production n'a pas de conséquences néfastes jusqu'au milieu des années 1970. Dans la seconde moitié des années 1970, le nouveau système productif basé sur la monoculture de riz révèle sa rigidité, son incapacité à répondre aux exigences de sécurité alimentaire villageoise que la détérioration des conditions économiques nationales menace et qu'amplifient les aléas climatiques ${ }^{16}$.

\begin{tabular}{|c|c|c|c|c|c|c|c|c|c|c|c|c|c|}
\hline \multicolumn{2}{|c|}{ Période moyenne } & Nov. & Déc. & Janv. & Février & Mars & Avr. & Mai & Juin & Juillet & Août & Sept. & Oct. \\
\hline \multicolumn{2}{|c|}{ Pluviométrie } & \multicolumn{3}{|c|}{$\begin{array}{c}\text { Petite Saison des } \\
\text { Pluies }\end{array}$} & $\begin{array}{l}\text { Petite Saison } \\
\text { Sèche }\end{array}$ & \multicolumn{3}{|c|}{ Grande saison des pluies } & \multicolumn{5}{|c|}{ Grande Saison Sèche } \\
\hline \multicolumn{2}{|r|}{ Hydrologie } & \multicolumn{5}{|c|}{$\begin{array}{l}\text { Etiage avec éventuellement crue-flash en } \\
\text { fin de petite saison des pluies }\end{array}$} & Début de crue & \multicolumn{2}{|c|}{$\begin{array}{c}\text { Pointe de crue } \\
\text { Extension maximale } \\
\text { des eaux }\end{array}$} & $\begin{array}{c}\text { Décrue } \\
\text { Début de } \\
\text { récession }\end{array}$ & $\begin{array}{c}\text { Décrue } \\
\text { Fin de } \\
\text { récession } \\
\end{array}$ & \multicolumn{2}{|c|}{ Etiage } \\
\hline \multirow{2}{*}{  } & $\begin{array}{c}\text { Dans la } \\
\text { plaine } \\
\text { inondable et } \\
\text { dans les } \\
\text { seuils fleuve- } \\
\text { lac }\end{array}$ & \multicolumn{5}{|c|}{ Opportunités de pêche réduites } & $\begin{array}{c}\text { Nasse fixe avec } \\
\text { ou sans } \\
\text { paradière sur } \\
\text { cheneaux de } \\
\text { circulation de } \\
\text { l'eau (vers la } \\
\text { plaine) }\end{array}$ & $\begin{array}{l}\text { Oppo } \\
\text { pêch }\end{array}$ & $\begin{array}{l}\text { iités de } \\
\text { duites }\end{array}$ & $\begin{array}{c}\text { Nasse fixe avec } \\
\text { ou sans } \\
\text { paradière sur } \\
\text { cheneaux de } \\
\text { circulation de } \\
\text { l'eau (vers le } \\
\text { fleuve) }\end{array}$ & $\begin{array}{l}\text { Paradière } \\
\text { mobile } \\
\text { (senne) et } \\
\text { panier dans } \\
\text { les mares } \\
\text { résiduelles }\end{array}$ & \multicolumn{2}{|c|}{$\begin{array}{l}\text { Opportunités de } \\
\text { pêche réduites }\end{array}$} \\
\hline & \begin{tabular}{|c} 
Sur les \\
platiers des \\
lacs
\end{tabular} & $\begin{array}{c}\text { Pêche } \\
\text { à la } \\
\text { battue }\end{array}$ & \multicolumn{9}{|c|}{ Pas de pêche } & \multicolumn{2}{|c|}{$\begin{array}{l}\text { Pêche à la } \\
\text { battue }\end{array}$} \\
\hline
\end{tabular}

Calendrier moyen et simplifié des pêches traditionnelles au milieu du XXe siècle (source : enquêtes au village de Kipo 2007-2010).

La crise vivrière induite par la conjonction de la villagisation et de la crise économique pouvait trouver sa résolution dans deux stratégies locales non-antagonistes ${ }^{17}$. La première était le retour à l'ancien terroir cultivé, la plaine inondable. La seconde était l'intensification des activités marchandes. Le retour vers la plaine inondable s'est fait progressivement dès le début des années 1980 et semble être achevé à la fin des années 1990. La stratégie adoptée a été de charger les anciens de la mise en culture progressive de la plaine inondable tandis que les autres générations assuraient le travail initial de défriche et continuaient parallèlement de conduire la riziculture inondée des dépressions argileuses de la terrasse ferralitique. Cependant, l'amélioration de la sécurité alimentaire ne pouvait être immédiate et l'intensification des activités marchandes s'imposait. Mais l'éventail de ces activités était réduit en raison de contraintes agronomiques et de la crise économique.

D'un point de vue agronomique, les anciennes stratégies de vente des surplus vivriers ou du coton n'étaient pas envisageables avant le réinvestissement de la plaine. D’autre part, les politiques nationales et la crise économique mondiale avaient changé les conditions d'échange. La villagisation, la réorganisation malheureuse des réseaux 


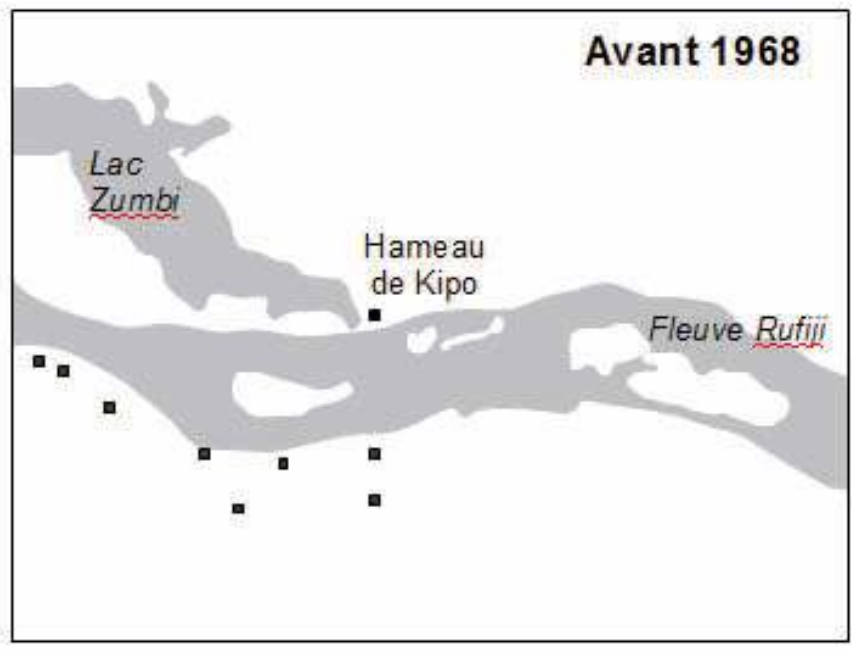

Carte 2

La villagisation de 1967-1968 à Kipo (Rufiji, Tanzanie).

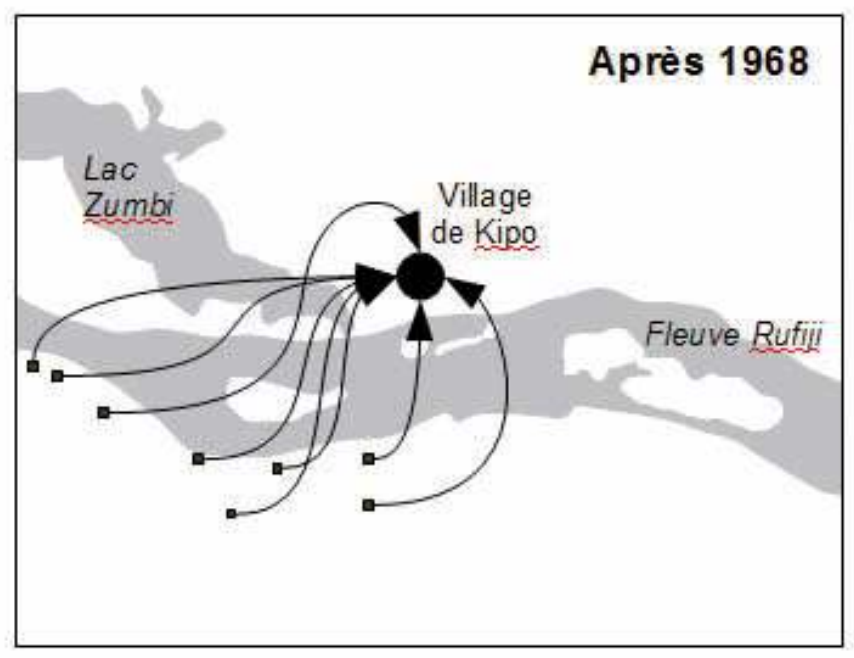

de commercialisation (Moshi, 1992 : 67) et la dégradation des prix mondiaux rendaient désormais peu attrayantes les cultures d'exportation (Maghimbi, 1992 : 219-220). La crise des années 1980 (Nindi, 1992 : 180) ${ }^{18}$ s'accompagna d'une réorientation générale du pôle monétaire de l'économie paysanne vers le vivrier et vers les activités extra-agricoles (Bantje, 1982 :i; Havnevik, 1993 : 194). L'enclavement de la partie occidentale de la plaine inondable du Rufiji et la péjoration consécutive des conditions d'échanges expliquent que l'option vivrière à Kipo était nécessairement limitée. Par contre, la proximité du lac, la possibilité de la pêche au filet et l'existence d'un marché urbain populaire pour le poisson fumé se révélèrent salutaires pour l'économie villageoise. La pêche devint rapidement le moyen local quasi-exclusif d'obtention du revenu. Mais pour répondre aux exigences de cette nouvelle fonction, cette activité devait être conduite toute l'année ce qui signifiait la généralisation des techniques au filet.

\section{Significations sociales des changements techniques}

La réflexion économique sur l'évolution de la place de la pêche au sein de l'économie villageoise a été menée considérant que l'agent économique était un villageois indifférencié, artefact commode d'une description sommaire de la manière dont l'évolution de l'environnement socio-économique avait pu peser sur l'évolution des stratégies halieutiques. Lorsque les habituelles sources de revenu monétaire se tarissent, le caractère très saisonnier de la pêche traditionnelle, malgré sa très bonne productivité journalière, la disqualifie comme solution de remplacement. A contrario, la pêche au filet, malgré une productivité journalière plus faible, offre le double avantage de l'émancipation de la saisonnalité et des lieux de pêche traditionnels, à la fois dispersés et désormais moins accessibles en raison de la villagisation. Elle rend possible l'exploitation tout au long de l'année des ressources halieutiques du lac Zumbi qui, de plus, sont désormais au voisinage immédiat des habitations. Il reste à comprendre comment cette nouvelle technique est socialement 


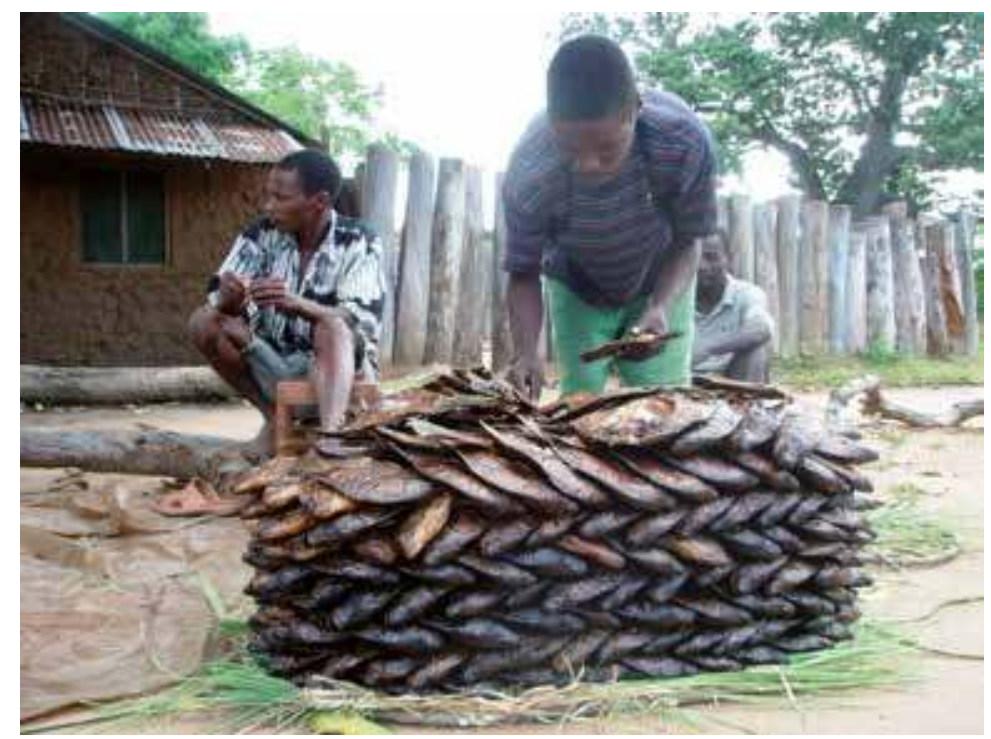

Les pêcheurs vendent la plus grosse partie (près de $88 \%$ ) de leur poisson frais à des revendeurs. Le poisson est ensuite fumé. Suivant le capital dont dispose le revendeur, il fume lui-même le poisson ou emploie un villageois. Une fois fumé, le poisson est soigneusement conditionné à l'aide de matériaux locaux. Finalement, le revendeur charge le poisson sur un bus et se rend en ville pour vendre son stock.
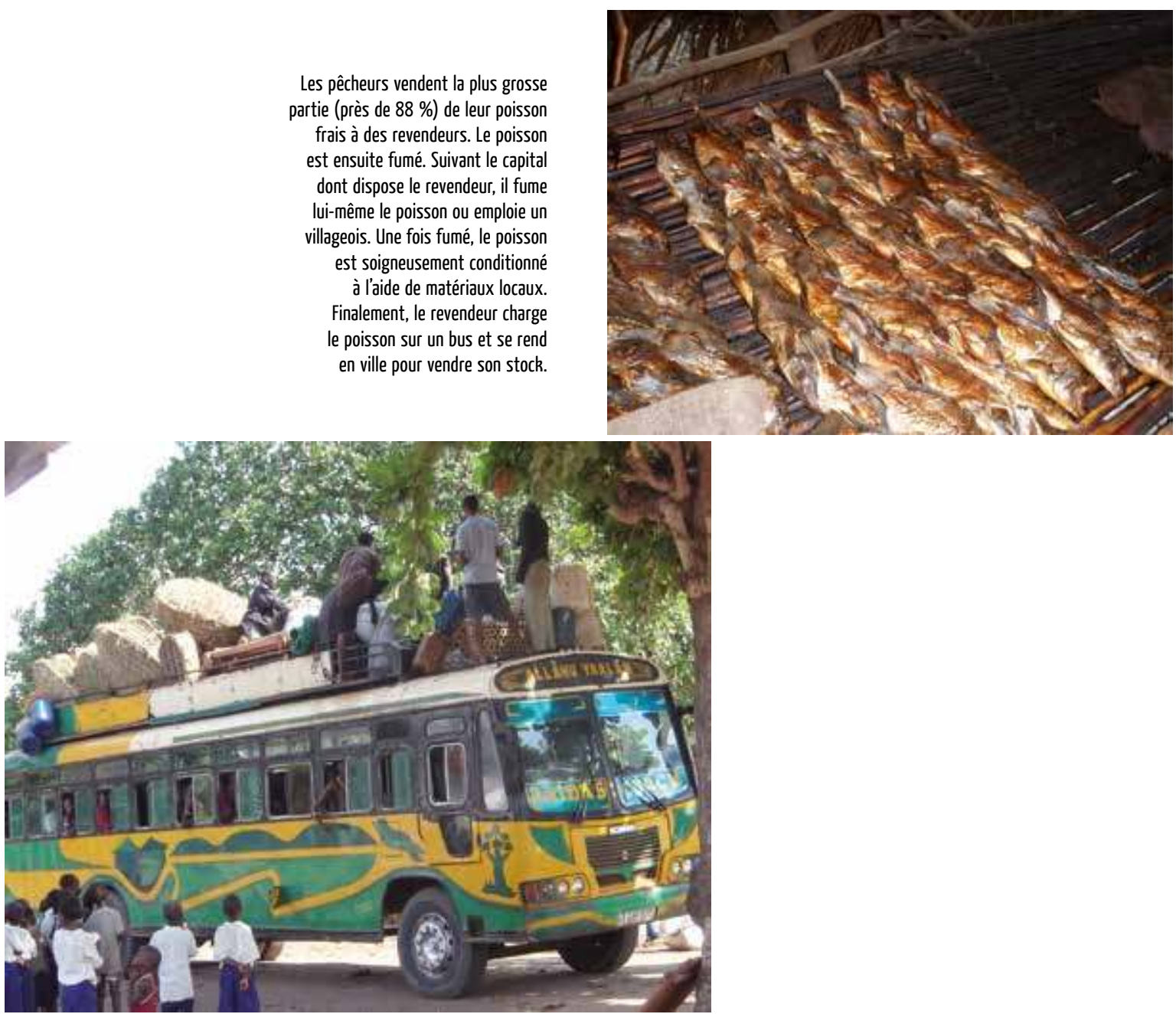
jamais la productivité telle que nous l'avons jusqu'ici envisagée. Ce que leurs récits expriment, c'est la frustration de jeunes gens face à ce qu'ils considéraient comme une injuste répartition du produit de la pêche au regard de leur participation effective. La pêche au filet leur offrait une voie d'émancipation du contrôle des aînés, d'appropriation immédiate du résultat de leur effort productif.

« On partageait les prises. Ils entassaient les poissons par taille. Nous étions assis comme maintenant et ils nous jetaient les poissons. Mais certains tas, ceux des gros poissons, on nous en privait (...) Tu es privé des plus gros poissons et pourtant pour travailler tu as travaillé! Tu as participé à la battue, même la nuit! (...) Tu es trop jeune. Tu regardes mais tu ne peux rien dire parce qu'il y avait Sungura Mpingi notre bête noire ${ }^{21}$ qui nous exploitait parce que nous étions trop jeunes (...) On n'avait pas de gros poissons, on nous donnait simplement ceux de petite taille ou les moyens (...)» (Omari Kindinda le 28 août 2009).

Après avoir eu des filets, les gens se dirent: Ah! En fait nous sommes exploités. Il vaut mieux que nous utilisions les filets (...) Désormais on pouvait pêcher à deux seulement et obtenir du poisson. On a laissé la pêche à la battue loin derrière nous et on a avancé (...) Les anciens, ils essayèrent un peu de nous retenir: Eh, les gars, si vous nous abandonnez, si vous ne participez plus à la pêche à la battue, nous allons mourir! On leur a répondu qu'on ne reviendrait pas (...) vous prenez les gros poissons et nous, on se retire définitivement! On est plus là, si vous les anciens vous voulez pêcher, pêchez, sinon tant pis (...) » (Halfani Kindinda le 28 août 2009).

« Les anciens n'auraient pas été abandonnés s'ils n’avaient pas cette mauvaise habitude. Ceux qui travaillent dur sont les jeunes, ils tirent les palissades, ils battent l'eau... Ce sont eux qui ont la force, mais voilà qu'une fois le poisson pris, on te donne de tout petits poissons et tandis qu'ils reçoivent les plus gros et les plus nombreux. Si on les interroge ils répondent qu'un jeune ne peut pas recevoir la même chose qu'un aîné. C'est ici qu'on a réfléchi. Si on n’agissait pas avec sagesse, ils se seraient fâchés (évocation de la sorcellerie). Qu'on leur dise, la pêche à la battue n'est pas suffisante, il nous semble mieux de nous tourner vers les filets, mais quand vous aurez besoin de nous, nous viendrons. Les aînés furent satisfaits mais en fait nous les laissions tomber. Une fois que ce fut fait. - La compagnie, aujourd'hui, allons à la pêche à la battue. Ah mon cher, il fait trop froid, on ne peut pas! C'est comme ça que nous leur avons échappé. » (Shabani Kindinda 28 août 2009).




Pour les jeunes Kindinda, l'utilisation des techniques de pêche au filet représente une opportunité d'appropriation directe du fruit de leur travail en échappant au contrôle des anciens. Mais cette stratégie s'oppose au principe central de l'organisation lignagère, le principe d'antériorité qui fonde l'autorité des aînés sur les cadets. Ce faisant le cycle viager est brisé: le devenir social auquel chaque personne aspire dans le cadre de l'idéologie domestique c'est l'aînesse dont le statut et la condition dépendent du rapport aîné/ cadet. La déstructuration de l'organisation lignagère et le déliement du rapport d'autorité ne sont évidemment pas le résultat de l'introduction de la pêche au filet. En Afrique de l'Est, la modernité, sous ses avatars économiques, politique et religieux nourrit son mouvement de la tension intergénérationnelle au moins depuis le début de la colonisation. Les auteurs s'accordent sur la contribution essentielle de l'exploitation de cette tensionlà au profit de la généralisation de l'économie de marché, de l'émergence de nouveaux niveaux de pouvoir liés à l'éducation scolaire, à la christianisation et au développement des partis politiques nationaux, comme le TANU (Illife, 1979; Lockwood, 1998; Pels, 1998; Sunseri, 2001).

Ladoption des techniques de pêche au filet s'appuie sur et nourrit la déliquescence du lien aîné/cadet, mais elle n'est qu'un élément conjoncturel du faisceau cohérent de facteurs qui imposent la modernisation et par conséquent la dislocation des communautés agricoles domestiques. Si les tensions intergénérationnelles s'avèrent être une ligne de faiblesse sur laquelle la modernisation joue efficacement, la pêche, pour les raisons que nous avons déjà évoquées, se prête plus aisément que l'agriculture à la marchandisation des rapports sociaux de production ${ }^{23}$. Avec cette marchandisation, on passe du temps cyclique et viager de la communauté agricole domestique au temps instantané et compulsif de la marchandise.

Ce passage est loin d'être abouti. Car si les structures lignagères et les institutions qui leur sont associées se désagrègent, c'est au profit d'institutions modernes dont l'existence formelle contraste avec la vacuité fonctionnelle. Tandis que les institutions traditionnelles disparaissent, la nécessité d'assurer certaines de leurs fonctions persiste ce dont les structures modernes mises en place (le parti - aujourd'hui disparu - et le conseil municipal à l'échelle villageoise, le district, etc. à une échelle plus petite) sont incapables. Les villageois continuent donc de dépendre de liens de solidarité et d'échange «traditionnels». En l'absence de toute sécurité sociale, les inévitables aléas de la vie économique et sociale doivent être compensés, bon an mal an, par des réseaux désormais non institutionnels. La morbidité ou l'accident hydro-climatique, par exemple, ont de très graves impacts sur l'activité productive du groupe commensal dans la mesure où celui-ci assure à peine, en temps normal, sa survie. Les stratégies qui sont alors mises en place ressortissent prioritairement de la pression à la solidarité (solidarité obligée) sur les réseaux de parenté et d'alliance conçus sur le mode de la communauté domestique et, dans, une moindre mesure, sur l'amitié. 


\section{$\&$}

Derrière la simplicité de la modernisation technique qu'illustrerait l'adoption des techniques de pêche au filet apparaît la complexité de la modernisation comme processus social, ici avatar contextualisé de la mondialisation marchande. De ce processus, les frères Kindinda saisissent évidemment l'aspect qui leur est le plus accessible, à savoir l'individualisation qu'ils appréhendent sur le mode de l'émancipation du contrôle des anciens. D'ailleurs, tandis que l'individualisation est effectivement avancée comme le révèle la disparition des institutions « traditionnelles », la destinée urbaine de la majorité des personnes nées au village ou encore la part croissante de la marchandise dans l'économie villageoise, d'autres manifestations classiquement associées à la modernisation restent d'une grande sobriété... Aujourd'hui, l'agriculteur du Rufiji va au champ muni d'une houe et d'un coutelas. Il a acheté le coutelas et le fer de la houe à la boutique du village. Il a lui-même confectionné le manche de la houe. Il est vêtu pour l'occasion d'un souvenir de chemise. Il y a un siècle et demi, il aurait été muni d'une houe et d'une herminette fabriquées par un voisin forgeron et vêtu, peut-être, d'une jupe d'écorce battue. La modernisation technique de la pêche, certes plus marquée, reste cependant modeste: elle se résume à l'arrivée des filets industriels. Les embarcations, le fumage et le conditionnement du poisson partant pour la ville continuent d'être réalisés à partir de matériaux locaux selon des techniques inchangées. Voilà la contribution de la modernité au développement des forces productives dans le Rufiji. Pour le reste, il faut chercher dans les musées ou chez les marchands d'arts premiers les magnifiques objets d'usage courant que produisaient naguère ces populations et qui sont désormais remplacés par de la vaisselle plastique ou aluminium de l'industrie de masse chinoise. Voilà la contribution de la modernité au confort domestique des habitants du Rufiji. À quel progrès financier ce piétinement des forces productives, dans le cadre concurrentiel de l'économie de marché, correspond-elle? Bien qu'on ne dispose d'aucune information sur l'évolution du revenu des habitants du Rufiji au cours du $x^{e}$ siècle, les indices pointent plutôt vers une stagnation. Ainsi, nos enquêtes révèlent que sur un demi-siècle, une récolte exceptionnelle se traduit toujours par le même niveau de consommation: l'achat d'une bicyclette ou d'un poste radiophonique...

En réponse à l'état d'intense précarité dans lequel les conditions d'échange plongent les villageois, l'individualisation des pratiques productives et la pression sur la ressource vont croissant. À la gestion communautaire des ressources halieutiques qui devrait logiquement s'imposer, s'opposent les stratégies individuelles de survie où la recherche de monétaire est une nécessité quotidienne à laquelle seule la pêche, bon an mal an, est susceptible de répondre. Or, les conditions d'échange se fondent sur des rapports de forces politiques dont on n'imagine guère qu'ils puissent évoluer favorablement (Duvail \& al., 2010). Pire, à moyen terme, la construction du barrage hydroélectrique de Stiegler's gorge (carte 1) pourrait sonner le glas de l'économie villageoise (Duvail et Hamerlynck, 2007; Hamerlynck \& al., 2010) et sceller tragiquement le sort des communautés villageoises du Rufiji.

Auteurs : Jean-Luc Paul (Université des Antilles - IMAf), Olivier Hamerlynck (National Museums of Kenya, KENWEB), Stéphanie Duvail (IRD - UMR PALOC) E Kassim H. Kindinda (membre de la communauté villageoise de Kipo). 


\section{NOTES}

Photo d'ouverture: Des villageois pêchent à la senne embarquée dans un bras du fleuve Rufiji créé par la crue. Toutes les photos sont des auteurs.

1. Pour la réduction de l'accès aux ressources naturelles dans le Rufiji en période coloniale voir Sunseri (2002).

2. Havnevik (1993) traitent, notamment, des stratégies de réaménagement de l'éventail des activités des populations du Rufiji en période postcoloniale.

3. Pallières et Paul (2013) offrent un exemple de l'analyse des mécanismes de cette involution dans le cas de la paysannerie d'une autre région africaine, celle de la Sella Limba (Sierra Leone).

4. En Afrique de l'Est, plusieurs travaux analysent la complexité des déterminants de l'évolution technique et organisationnelle des pêcheries traditionnelles. Au Malawi par exemple, Nakayama (2008) met en évidence l'importance des émigrants urbains de retour au village tandis que Russell et Dobson (2011) analysent le rôle des autorités traditionnelles.

5. La pêche (illégale) dans la Réserve du Selous est exclusivement pratiquée au filet.

6. La taille du filet est un indicateur du coût de celui-ci. Étant donné la très faible capacité d'investissement des villageois, cet élément est à prendre en compte dans l'analyse des stratégies halieutiques.

7. Ou une brasse, c'est-à-dire l'envergure des bras.

8. Les Citharinus congicus actuellement pêchés dans les lacs de la vallée inondable du Rufiji (hors Selous) ont une taille moyenne de 19,3 cm. La taille adulte de ce poisson est de $50 \mathrm{~cm}$.

9. L'aide alimentaire était distribuée au chef-lieu du district, Utete, à une journée de marche de Kipo.

10. Les lacs occupent d'anciens lits de rivière, ils sont de forme allongée. Les platiers occupent les deux extrémités de la longueur et les extrémités des éventuelles digitations. Avant l'introduction du filet, les lacs sont très peu concernés par la pêche. En 1937, Barker les considère exempts d'activités humaines (Barker, 1937).

11. L'observation des groupes de pêche à la nasse fixe actuellement recensés au village de Kipo rend cette évaluation vraisemblable. Bien qu'il n'y ait pas de battue et que les paradières soient relativement courtes, l'installation et l'exploitation de ces dispositifs sont assurées par des groupes de 9 à 12 personnes.

12. On compare ici les techniques mises en œuvre dans les zones de pêches légales. Dans la réserve du Selous, c'est l'abondance de la ressource halieutique qui explique la productivité élevée et non la technique utilisée (voir le tableau 3).

13. À ce propos voir Havnevik, 1993.

14. La période de soudure sépare le moment où s'épuisent les stocks issus de la récolte précédente de la prochaine récolte.

15. La villagisation de l'ensemble du pays ne sera entreprise qu'à partir de 1974, avec l'appui de la Banque Mondiale. Il s'agit d'accroître le contrôle étatique sur la production agricole, d'intensifier l'insertion de l'agriculture au marché international afin de faciliter la captation d'un surplus paysan par l'État (Lockwood, $1998: 81)$.

16. La dégradation des conditions d'échange pour les villageois résulte des difficultés de commercialisation du riz qu'entraîne le contrôle croissant de l'État et du mauvais approvisionnement des boutiques coopératives. Le hiatus entre le prix d'achat des denrées sur le marché noir et le prix de vente du riz sur le marché officiel s'élargit. Le cours au marché noir du shilling tanzanien l'illustre: au début 1986 le taux de change y est plus de huit fois supérieur au taux officiel (Kaufmann \& Connell, 1997).

17. L'émigration vers la ville est une troisième stratégie. L'analyse de l'évolution démographique du village de Kipo démontre que l'émigration y est importante et continue.

18. La situation tanzanienne n'est pas originale: en Afrique subsaharienne, le revenu par habitant diminue de 1,2\% par an durant les années 1980 (Nations Unies 2000)

19. Les informations utilisées pour la présente reconstitution historique sont issues d'entretiens avec des villageois entre novembre 2006 et août 2010.

20. Un pêcheur allochtone s'associe avec un pêcheur local qui dirige la pirogue. Aucun pêcheur du village ne maîtrisait, en 2010, la technique de l'épervier mais nous avons observé plusieurs d'entre eux s'entraînant au lancer du filet.

21. Le terme ici utilisé est kiboko, qui signifie « hippopotame » mais qui désigne également le coup de nerf d'hippopotame que recevait à l'époque le contrevenant à l'ordre colonial. 
22. Dans la société agricole domestique, il existe un cycle de vie normatif qui représente les étapes idéales par lesquelles devrait passer une personne, de sa conception jusqu'à sa mort. La norme diffère d'un genre à l'autre mais, à l'intérieur d'un même genre elle reste la même d'une personne à l'autre (voir Meillassoux 1975).
23. Par exemple, l'accès au monétaire via la plantation d'anacardiers ne peut pas répondre aux besoins d'émancipation des cadets: ils ne maîtrisent pas l'accès au foncier, le délai entre la plantation et la première récolte est trop long et le contrôle par les aînés du produit est plus facile que dans le cas de la pêche.

\section{POUR CITER CET ARTICLE}

Paul, J.-L., Hamerlynck, O., Duvail, S. \& Kindinda Kassim H. In Dupré, L., Lasseur, J. \& R. Poccard-Chapuis Pâturages Techniques \& Culture 63: 234-257

\section{RÉFÉRENCES}

Bantje, H., Mrisho, F. \& B. Ljungqvist 1979 A Nutrition baseline survey in four villages in the lower Rufiji Valley. Dar-es-Salaam: Bralup Research Paper 55.

Bantje, H. 1982 Seasonality and response: some observations on seasonal variations in the Rufiji valley. Dar-esSalaam: Bralup Service Paper 82/4.

Barker, R. 1937 The Rufiji River, Tanganyika Notes and Records 4 : 15-16.

Biermann, W. 1998 The Tanzanian economy 1920-1985 : colonial valorisation, reconstruction, and crisis. Dar-esSalaam: Dar-es-Salaam University Press.

Bigsten, A. \& Danielson, A. 2001 Tanzania : is the ugly duckling finally growing up? Uppsala: NordiskaAfrikainstitutet Research report no 120.

Blanc-Pamard, C. \& Milleville, P. 1985 Pratiques paysannes, perception du milieu et système agraire. In A travers champs, agronomes et géographes. Paris : ORSTOM (Dynamique des systèmes agraires) : 101-138.

Bryceson, F.B. 1996 Liberalizing tanzania's food trade: public \& private faces of urban marketing policy, 19391988. Londres : James Currey Publishers.

Cochet, H. 2011 L' Agriculture comparée.Versailles : Éditions Quae.

Dufumier, M. 1985 Systèmes de production et développement agricole dans le Tiers-Monde, Les Cahiers de la Recherche-Développement (6) : 31-38.

Duvail, S. \& Hamerlynck, O. 2007 The Rufiji River flood: plague or blessing? International journal of biometeorology 52 (1): 33-42.

Duvail, S., Médard C. \& Paul, J.-L. 2010 Les Communautés locales face aux grands projets d'aménagement des zones humides côtières en Afrique de L'Est, Politique Africaine 117: 149-172.

Duvail, S. \& al. 2013 Jointly thinking the post-dam future : exchange of local and scientific knowledge on the lakes of the Lower Rufiji, Tanzania, Hydrological Sciences Journal 59 (3-4) : 713-730.

Hamerlynck, O., Nyingi, D.W., Paul, J.-L. \& Duvail, S. (sous presse) Fish-based farming systems: maintaining ecosystem health and flexible livelihood portfolios. In African farming systems. CGIAR, World Bank. Earthscan.

Hamerlynck O., \& al. 2010 The Large-scale irrigation potential of the Lower Rufiji Floodplain (Tanzania): reality or persistent myth?. In B.Calas \& al. (Dir.) Shared waters, shared opportunities. Nairobi: IFRA and Hakimani College: 219-234.

— 2011 To Connect or not to connect? Floods, fisheries and livelihoods in the Lower Rufiji floodplain lakes, Tanzania, Hydrological Sciences Journal 56 (8) : 1436-1451. 
Havnevik, K.J., 1993 Tanzanian : the limits to development from above. Upssala-Stockholm: NordiskaAfrikainstitutet.

Hopson, A.J., 1979 Report on the freshwater fisheris on the lower Rufiji River with particular reference to possible changes resulting from modifications to the environment by the proposed dam at Stiegler's Gorge. Rome: FAO Project Technical Paper $\mathrm{N}^{\circ} 1$.

Iliffe, J., 1979 A Modern history of Tanganyika. Cambridge: Cambridge University Press.

Kaufmann D. \& S.A. O'Connell 1997 The Macroeconomics and delayed exchange rate unification: theory and evidence from Tanzania. In M. Kiguel, J.-S. Lizondo \& S.A. O'Connell (Dir.) Parallel exchange rates in developing countries. London: Macmillan.

Lockwood M., 1998 Fertility and household labour in Tanzania: demography, economy, and society in Rufiji District, c.1870-1986. Oxford \& New-York: Clarendon Press.

Maghimbi, S. 1992 The Abolition of peasant cooperatives and the crisis in the rural economy in Tanzania . In P.G. Forster \& S. Maghimbi (Dir.) The Tanzanian peasantry: economy in crisis. Aldershot: Avebury: 216-235.

Meillassoux, C. 1975 Femmes, greniers et capitaux. Paris: Maspéro.

Milleville, P. 1974 Enquête sur les facteurs de la production arachidière dans trois terroirs de moyenne Casamance, Cahiers ORSTOM (Série Biologie 24) : 65-97.

- 1976 Comportement technique sur une parcelle de cotonnier au Sénégal, Cahiers ORSTOM, (Série Biologie 26) : 263-275.

Moshi, H.P.B. 1992 Cooperative and agricultural development problems and policy options: the Tanzanian experience 1961-1990. In Forster, Peter G. and Sam Maghimbi (Dir.) The Tanzanian Peasantry: Economy in Crisis. Aldershot: Avebury: 58-73.

Mpangala, G. 1996 The Future of co-operatives in the development of the peasant sector in Tanzania. In D. Schmied (Dir.) Changing Rural Structures in Tanzania. Münster: Lit Verlag: 49-55.

Mvungi S. \& H. Mwakyembe, 1996 The Regulation of land tenure (established villages) Act, 1992 and its implications for customary land rights in Tanzania. In D. Schmied (Dir.) Changing rural structures in Tanzania. Münster: Lit Verlag: 73-84.

Nations Unies, 2000 Nous, les peuples - Le rôle des Nations Unies au xxie siècle. New-York: Nations Unies.

Nakayama, S. 2008 City lights emblaze village fishing grounds: the re-imagining of waterscape by lake Malawi fishers, Journal of Southern African Studies 34 (4): 803-821.

Nindi B., 1992 Agricultural marketing reforms and the public versus private debate in Tanzania. In S. Maghimbi and P. Forster (Dir.) The Tanzanian peasantry: economy in crisis. Aldershot: Avebury: $169-189$.

Pallière, A. \& Paul, J-L. 2013 Marchandisation du travail et différenciation sociale en Sella Limba (Sierra Leone) de 1950 à nos jours, Journal des Africanistes 83 (2) : 116-141.

Paul, J-L., Bory, A., Garganta, E. \& Fabri, A. 1994 Quel système de référence pour la prise en compte de la rationalité de l'agriculteur : du système de production agricole au système d'activité, Les Cahiers de la Recherche-Développement (39) : 7-19.

Paul J.-L., S. Duvail \& Hamerlynck,O. 2011 Appropriation des ressources «naturelles» et criminalisation des communautés paysannes - Le cas du Rufiji, Tanzanie, Civilisations 60 (1) : 143-175.

Pels P., 1998 A Politics of presence: contacts between missionaries and Waluguru in late colonial Tanganyika. Amsterdam: Harwood Academic Publishers (Studies in Anthropology and History 22).

Russel, A.J.M. \& T. Dobson 2011 Chiefs as critical partners for decentralized governance of fisheries: an analysis of co-management case studies in malawi, societies and natural resources: An International Journal 24 (1): 734-750.

Sunseri T., 2001 Vilimani: Labor migration and rural change in early colonial Tanzania, 1884-1914. Portsmouth, N. H. : Heinemann.

Sundet G., 1996 The Politics of land tenure in tanzania: the modernising state vs. smallholder farmers. In D. Schmied (Dir.) Changing Rural Structures in Tanzania. Münster: Lit Verlag: 57-72. 


\section{RÉSUMÉ}

De la nasse au filet: stratégies halieutiques et modernisation dans le Rufiji (Tanzanie). Depuis plus d'un siècle, l'économie des villages de la plaine inondable du Rufiji (Tanzanie) est fondée sur la complémentarité des activités agricoles et des activités halieutiques. À partir de la fin des années 1970, les pêches traditionnelles à la nasse, pratiquées à pied saisonnièrement lors de la crue annuelle, sont supplantées par les pêches embarquées (pirogues monoxyles) au filet dans les lacs, pratiquées elles toute l'année. Larticle s'interroge sur les déterminants de cette substitution. Après avoir rejeté l'hypothèse techniciste vulgaire qui voudrait que l'innovation technique soit synonyme de progrès, la pertinence du critère de productivité des différentes techniques de pêche est examinée à la lumière de l'évolution de la place des activités halieutiques dans l'économie villageoise. Il apparaît qu'à partir de cette époque, l'évolution économique nationale et internationale prive les villageois de leurs principales ressources monétaires habituelles. En l'absence de toute alternative, la pêche s'impose alors comme unique source de revenu monétaire, une fonction qu'elle ne peut remplir qu'à condition d'être pratiquée tout au long de l'année, ce que seules les techniques au filet permettent. Cependant, les représentations villageoises des causes de la généralisation de la pêche au filet sont différentes. Pour ceux d'entre eux, aujourd'hui très âgés, qui furent les pionniers de cette innovation, c'est le souci d'émancipation des cadets vis-à-vis des aînés qui aurait été le moteur de l'adoption de techniques de pêche au filet. Ce point de vue entre en résonance avec les déterminations économiques à condition de considérer le processus de modernisation comme manifestation multiforme contextualisée de la mondialisation marchande.

\section{ABSTRACT}

From barriers to nets: fisheries strategies and modernisation in Rufiji (Tanzania) For over a century, the complementary nature of fishing and farming activities in the floodplain of the Rufiji River (Tanzania) has formed the basis of the economy in its villages. Starting in the 1970s, the fishery evolved from a seasonal activity practiced on foot during flooding and using traditional traps to year-round dugout canoe operated lake fisheries using nets. The paper analyses the drivers of this change. After having rejected the simplistic technocratic hypothesis that technical innovation equals progress, the paper examines the relevance of the productivity of the different fishing techniques in the light of the evolution of the role of fisheries in the village economy. During the change-over period, the national and international economy deprives the villages of their main habitual sources of cash income. In the absence of any alternative, fisheries imposed themselves as the unique source of cash income, a function they can only fulfil if practiced year-round and this can only be achieved using canoes and nets. However, the perception of the causes of these changes in the villages is differentiated. For those who initiated the change-over, and who are now of an advanced age, the main driver of the transition to nets was the empowerment of the youth versus the elders. Such an interpretation resonates well with the economic determinants. However, it requires considering the modernisation process as a contextualised multi-sided expression of market globalisation.

\section{MOTS-CLÉS}

pêche, Rufiji, Tanzanie, innovation technique

\section{KEYWORDS}

fishery, Rufiji, Tanzania, technical innovation 